\title{
Early hormone replacement therapy may yield benefits, researchers say
}

A lmost a decade after a blockbuster study linked the use of hormone replacement therapy (HRT) to higher dementia rates, mental health researchers say those concerns may have been overblown and clinicians may be missing an opportunity to safely improve cognitive functioning and alleviate symptoms of menopause in some women.

"You've seen, like I have, this pendulum swinging. Is HRT a risk factor for Alzheimer's? Do we believe the media in 2002 saying that if you get it you're also going to get dementia? Or do we believe the older and more recent data suggesting it's protective against cognitive decline?" Pauline Maki, associate professor in the departments of Psychiatry and Psychology at the University of Illinois at Chicago told delegates to a "Women in Mind" conference in Ottawa, Ontario, on Oct. 21. "I would argue that it depends on the timing of the initiation of treatment, the age of the patient and what kind of hormone therapy is being used."

Results of a Women's Health Initiative substudy on the incidence of dementia and mild cognitive impairment in postmenopausal women found that an additional 23 cases of dementia per 10000 per year would result from the use of estrogen plus progestin (JAMA 2003;289[20]:2651-62).

But Maki and others argued that those findings cannot be generalized to younger women and further research is needed to appropriately target use of the controversial treatment.

Although the study indicated that exposure to combined conjugated equine estrogen and medroxyprogesterone acetate doubled older women's risk for dementia, a similar increase was not shown for estrogen therapy alone, and meta-analyses suggest women exposed to HRT have an overall reduced risk of Alzheimer disease, Maki explained.

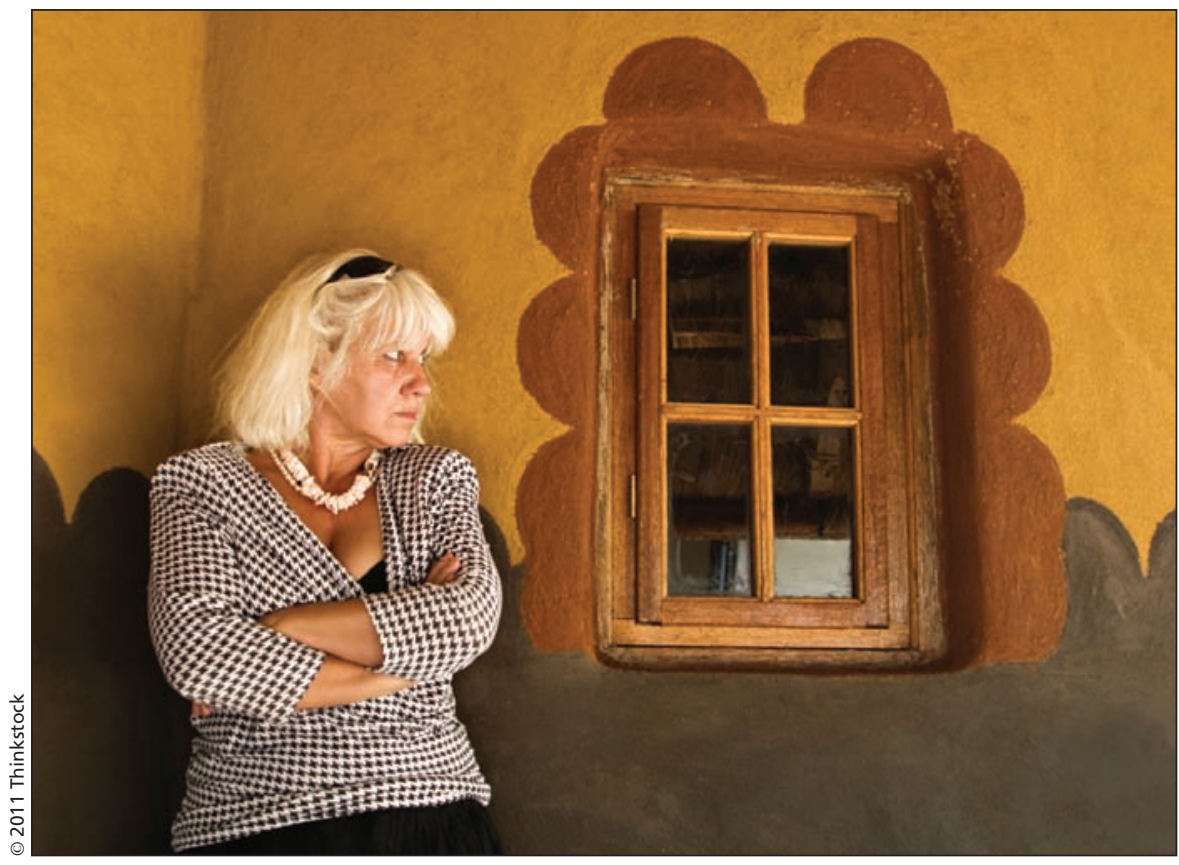

There may be a "window of opportunity" for the targeted use of hormone replacement therapy in younger, healthy women, argues Dr. Claudio Soares, an associate professor in the Departments of Psychiatry and Behavioural Neurosciences, and Obstetrics and Gynecology, at McMaster University in Hamilton, Ontario.

Recent research also indicates initiation of HRT early in the transition to menopause is associated with cognitive benefit, she added. "Women who initiate hormone therapy before their final menstrual period show increased blood flow to the hippocampus and better verbal memory compared to nonusers."

While exposure to estrogen doesn't improve memory beyond the baseline, it has been shown to prevent cognitive decay in women under 65 - one of the most sensitive indicators of risk for dementia, Maki said.

This suggests a "window of opportunity" for the targeted use of HRT in younger, healthy women, said Dr. Claudio Soares, an associate professor in the Departments of Psychiatry and Behavioural Neurosciences, and Obstetrics and Gynecology, at McMaster University in Hamilton, Ont. "We cannot say that one size fits all, so it's important that we start thinking about the critical timing in which to use estrogen, looking at different estrogen preparations and making decisions tailored to our patients needs."

Such a window could open onto new pathways for the treatment of depression at midlife because the period in which HRT presents the lowest risk while yielding the greatest cognitive benefit overlaps with a time when women are at a nearly twofold risk of depression as they make the transition to menopause, Soares added.

"Hormone intervention can be very helpful for patients with mood disorders in terms of helping them to stabilize and stay stable with or without antidepressants," he said. "While antidepressants and behavioural strategies are still the treatment of choice for depression across the lifecycle, it's important to know that hormonal strategies may have a role maintaining mood to augment those strategies." 
Further research is required to parse the broader health implications of targeted HRT use, Soares said, adding that the legacy of the Women's Health Initiative study has been a "huge lag" in research related to hormonal interventions. "There was a huge backlash in terms of funding and public reception. Recruiting patients for studies involving hormonal interventions after 2002 became a nightmare because people were scared of anything that had the word hormone it."

Patients "felt betrayed" when the health risks of HRT surfaced so "the process of getting them to at very least assess the risks and benefits [in light of new evidence] has become a double challenge," he explained.

Moving forward, physicians cannot "shy away" from that discussion, Soares added. "It's absolutely time we put this back on the table." - Lauren Vogel, CMAJ

CMAJ 2011. DOI:10.1503/cmaj.109-4041 